\title{
Neutron Activation Analysis: A Primary (Ratio) Method to Determine SI-Traceable Values of Element Content in Complex Samples
}

\author{
Peter Bode ${ }^{\star a}$, Robert R. Greenberg ${ }^{b}$, and Elisabete A. De Nadai Fernandes ${ }^{c}$
}

\begin{abstract}
The metrological principles of neutron activation analysis are discussed. It has been demonstrated that this method can provide elemental amount of substance with values fully traceable to the SI. The method has been used by several laboratories worldwide in a number of CCQM key comparisons - interlaboratory comparison tests at the highest metrological level - supplying results equivalent to values from other methods for elemental or isotopic analysis in complex samples without the need to perform chemical destruction and dissolution of these samples. The CCQM accepted therefore in April 2007 the claim that neutron activation analysis should have the similar status as the methods originally listed by the CCQM as 'primary methods of measurement'. Analytical characteristics and scope of application are given.
\end{abstract}

Keywords: Metrology · Neutron activation analysis · SI traceability · Uncertainty

\section{Introduction}

Neutron activation analysis is an isotopespecific analytical technique for the qualitative and quantitative determination of elemental content.[1] The method is based upon the conversion of stable atomic nuclei into radioactive nuclei by irradiation with neutrons and the subsequent detection of the gamma radiation emitted during the decay of these radioactive nuclei. Activation by neutrons may result in radionuclides from all elements (that have radioactive daughter products) present in the sample, with sometimes strongly different production rates. This mixture can be analysed in two different ways:

\footnotetext{
${ }^{\star}$ Correspondence: Dr. P. Bode ${ }^{a}$

Tel.: +31152783530

Fax: +31152783906

E-Mail: p.bode@tudelft.nl

aDelft University of Technology

Faculty of Applied Sciences

Department for Radiation, Radionuclides and

Reactors

Mekelweg 15

2629JB Delft, The Netherlands

bNational Institute of Standards and Technology NIST

Analytical Chemistry Division

100 Bureau Drive, Stop 8395

Gaithersburg, MD 20899-8395, USA

'Universidade de São Paulo

Centro de Energia Nuclear na Agricultura

Laboratório de Radioisótopos

Avenida Centenário, 303

13416-000 Piracicaba SP, Brazil
}

i) The resulting radioactive sample is chemically decomposed, and the elements are chemically separated: Destructive or Radiochemical Neutron Activation Analysis. This form of NAA will not be discussed here.

ii) The resulting radioactive sample is kept intact, and the radionuclides are determined, taking advantage of the differences in decay rates via measurements at different decay intervals: Nondestructive or Instrumental Neutron Activation Analysis (INAA).

The metrological basis for NAA was firmly established by the mid-to-late $1990 s,{ }^{[2]}$ although the fundamental research was largely completed earlier.

In 1996, the CCQM (Consultative Committee for Amount of Substance Metrology in Chemistry) identified that "...Strictly, traceability to the SI in measurements of amount of substance - or of any other quantity - requires that the measurements be made using a primary method of measurement, which is correctly applied and stated with an evaluated uncertainty...", and in 1998 the CCQM defined the concept of a primary method. ${ }^{[3-6]}$ A major advantage of primary methods is therefore their demonstrated straightforward traceability of the value of a measurand to the SI, requiring a complete evaluation and quantification of the uncertainty of measurement. Although not explicitly stated in the definition, it is implied by the "highest metrological properties" portion of the definition that the application of the method results in a small value of the measurement uncertainty that overlaps the conventional true value of the measurand (with the probability expected from the coverage factor applied in calculating the expanded uncertainty).

The authors demonstrated in the Inorganic Analysis Working Group of the CCQM already in 2000 that NAA based on the comparator method has the potential to fulfill the requirements of a primary ratio method with evidence of the methods' metrological fundamentals including the measurement equation, the evaluation and quantification of all sources of uncertainty and the aspects of traceability. Moreover, the performance of several laboratories worldwide in applying NAA in CCQM pilot studies and key comparisons (of minor and trace elements in complex matrices) between 2000 and 2007 was shown to be equivalent to those of laboratories applying ID-MS, one of the hitherto proclaimed primary methods. ${ }^{[7,8]}$ The CCQM, in its meeting of April 19, 2007, decided subsequently "...that NAA had claims to a similar status to that of the five methods listed originally by the CCQM and that NAA will be added to that list....". [9] The CCQM has decided to revisit the whole issue of primary methods in the future.

The analytical characteristics of INAA are:

- Non-destructive analysis. The testportion does not have to be converted into a solution, thus dissolution losses do not exist.

- The method is based upon processes in the atomic nucleus. The chemical form 
and physical state of the elements do not influence the activation and decay process.

- The method can be applied in a selfvalidating manner. A unique combination exists of the gamma-ray energies, their intensity ratios and the half-lives of the radionuclides, which provides opportunities for consistency checking.

- Many adjustable experimental parameters, sometimes over several orders of magnitude, allow the experimental design to be optimised and potential problems identified.

- Elements such as C, H, N, O and Si, often found as major components of many matrices commonly analyzed by NAA, do not activate well and contribute little or no gamma-ray activity.

- The method is suitable for determination of masses in the order of $10^{-6}-10^{-9} \mathrm{~g}$ and less, depending on the element to be determined.

\section{Metrology}

The induced radioactivity results in emission of gamma-radiation that is measured with a gamma-ray detector. This results in peak areas, $C$, in the gamma-ray spectrum, from which the decay corrected gamma-ray emission rate, $A_{0, i}$, of the activation product is determined at the end of irradiation (Eqn. (1)):

$$
\begin{aligned}
A_{0, i}= & \frac{\lambda_{i} \cdot C \cdot e^{\lambda_{i} \cdot t_{d}}}{\left(1-e^{-\lambda_{i} \cdot t_{i r}}\right) \cdot\left(1-e^{-\lambda_{i} \cdot t_{m}}\right)} \cdot f_{p} \cdot f_{l t c} \\
& =N_{i} \cdot<\sigma>\cdot \phi \cdot \Gamma \cdot \varepsilon
\end{aligned}
$$

$A_{0, i}=$ decay corrected gamma-ray emission rate of radionuclide $\mathrm{i} ; \lambda_{i}=$ decay constant $=(\ln 2) / t_{i 1 / 2}$ with $t_{i 1 / 2}$ the half life of the radionuclide; $C=$ net counts in $\gamma$-ray peak at energy of interest $\left(E_{\gamma}\right) ; f_{\mathrm{p}}=$ pulse pileup correction; $f_{\text {Itc }}=$ live time extension correction; $t_{\text {irr }}=$ irradiation time; $t_{\mathrm{d}}=$ decay time to start of count; $t_{\mathrm{m}}=$ live time of count; $N_{\mathrm{i}}$ $=$ number of atoms of target nuclide $\mathrm{i} ; \phi$ $=$ neutron fluence rate during irradiation; $\langle\sigma\rangle=$ neutron spectrum averaged cross section; $\Gamma=$ fraction of decays producing $\gamma$-ray of $E_{\mathrm{x}} ; \varepsilon=$ the full energy photo peak efficiency of the detector, i.e. the probability that an emitted photon of energy $E_{\mathrm{x}}$ will be detected and contribute to the corresponding photo peak in the spectrum.

As the mass of an element $m(\mathrm{E})$ follows from (Eqn. (2))

$$
m(\mathrm{E})=\frac{N_{i} \cdot M(\mathrm{E})}{N_{A} \cdot x\left({ }^{i} \mathrm{E}\right)}
$$

with $N_{\mathrm{A}}=$ Avogadro's number; $M(\mathrm{E})=$ molar mass of the element $\mathrm{E}$

$M(\mathrm{E})=\sum_{i} x\left({ }^{i} \mathrm{E}\right) \cdot M\left({ }^{i} \mathrm{E}\right)$

whereby $M\left({ }^{i} \mathrm{E}\right)$ is the molar mass of isotope $\mathrm{i}$ in element $\mathrm{E} ; x\left({ }^{i} \mathrm{E}\right)=$ isotopic abundance of the (stable) target isotope $\mathrm{i}$ of Element $\mathrm{E}$ :

$$
x\left({ }^{i} \mathrm{E}\right)=\frac{n\left({ }^{i} \mathrm{E}\right)}{\sum_{j} n\left({ }^{j} \mathrm{E}\right)}
$$

with $j$ as all stable isotopes of an element $\mathrm{E}$, the measurement equation in NAA can be derived straightforwardly from these equations, resulting in a linear relationship between the mass of the irradiated element $m(\mathrm{E})$ and the net counts in the photo peak (Eqn. (3)):

$$
m(\mathrm{E})=C \cdot \frac{\lambda_{i}}{\left(1-e^{-\lambda_{i} \cdot t_{i r}}\right) \cdot e^{-\lambda_{i} \cdot t_{d}} \cdot\left(1-e^{-\lambda_{i} \cdot t_{m}}\right) \cdot \phi \cdot<\sigma>\cdot \Gamma \cdot \varepsilon} \cdot f_{p} \cdot f_{l t c} \cdot \frac{M(\mathrm{E})}{x\left({ }^{i} \mathrm{E}\right) \cdot N_{A}}
$$

Eqn. (3) includes physical constants that are either tabulated (e.g. the decay constant, the fraction of decays resulting in a gamma-ray, the atomic mass); experimental parameters (e.g. the neutron fluence rate, the detector's efficiency and the pulse pile up and live time extension correction) and measured quantities (e.g. net counts in the peak and duration of irradiation, decay and counting).

Several of these constants and parameters are difficult to determine with small uncertainties. The comparator method is therefore preferred for assays at the highest metrological level where a sample and a standard of the element of interest are irradiated simultaneously, or under very similar conditions, and their radioactivities are determined consecutively on the same counting system. The ratio of the gamma-ray emission rates is a direct measure of the ratio of target nuclei in sample and standard, and thus of the ratio of the masses.

The ratios are corrected for differences between unknown and standard in isotope abundances $\left(R_{\mathrm{x}}\right)$, neutron fluence exposures $\left(R_{\phi}\right)$, effective cross sections $\left(R_{\sigma}\right)$ and differences in counting efficiencies $\left(R_{\varepsilon}\right)$. In most cases these ratios are extremely close to unity. The mass of the element of interest follows then from Eqn. (4):

$$
\begin{aligned}
m_{u n k}(\mathrm{E})= & m_{s t d}(\mathrm{E}) \cdot \frac{A_{0, u n k}}{A_{0, s t d}} \\
& \cdot R_{x} \cdot R_{\phi} \cdot R_{\sigma} \cdot R_{\varepsilon}-\text { blank }
\end{aligned}
$$

with $m_{\text {unk }}(\mathrm{E})=$ mass of an element $\mathrm{E}$ in the unknown sample; $m_{\text {std }}(\mathrm{E})=$ mass of an element $\mathrm{E}$ in the comparator standard; $A_{0, \mathrm{i}}=$ decay corrected gamma-ray emission rate, derived from the net photo peak area of unknown sample or standard (see Eqn. (1)); $R_{\mathrm{x}}=$ ratio of isotopic abundances for unknown and standard

$R_{x}=\frac{x\left({ }^{i} \mathrm{E}\right)}{x_{s t d}\left({ }^{i} \mathrm{E}\right)}$

$R_{\phi}=$ ratio of neutron fluences (including fluence drop off, self shielding, and scattering); $R_{\sigma}=$ ratio of effective cross sections if neutron spectrum shape differs from unknown to standard; $R_{\varepsilon}=$ ratio of counting efficiencies (differences due to geometry and $\gamma$-ray self shielding).

The uncertainties in all parameters in the simplified measurement equation (Eqn. (4)) can be evaluated and quantified. ${ }^{[10-12]}$ NAA determination of elemental mass can be made traceable to the SI because all parameters in the measurement equation are SI (or SI-derived) units such as $\mathrm{kg}, \mathrm{s}, \mathrm{m}$, mole and Becquerel, or dimensionless ratios.

\section{Scope of Use of NAA}

Obviously, the element of interest and sample matrix should have specific chemical properties, physical forms and physical characteristics for analysis by NAA. The activation rate and half-lives and energies of the gamma-ray emission of the radionuclides are decisive factors for selecting NAA. The very low $\mathrm{Z}$ elements (like $\mathrm{H}, \mathrm{He}, \mathrm{B}, \mathrm{Be}, \mathrm{C}, \mathrm{N}, \mathrm{O}$ ) are not suitable for determination by (thermal neutron) NAA, as are a few other elements like $\mathrm{Tl}, \mathrm{Pb}$ and $\mathrm{Bi}$.

Sample matrices of high density that contain significant fractions of high atomic number elements (both affecting gammaray self attenuation) are not good candidates for NAA measurements. Similarly, matrices that have extremely high neutron absorbing properties are difficult to measure accurately due to neutron self attenuation. Significant mass fractions of B, Li and $\mathrm{U}$ are also undesirable since their neutron capture results in the emission of chargedparticle radiation, which may cause excessive thermal heating during the irradiation.

Examples of measurements where the analytical characteristics of neutron activation analysis are employed at full advantage include:

- Solid materials that are difficult to dissolve completely, such as soils, rocks, minerals, air particulate matter, new 
composite materials, and materials with $\mathrm{C}, \mathrm{H}, \mathrm{N}, \mathrm{O}$ as major elements like biological material and plastics.

- Solid materials that are easily contaminated during preparation of the test portion if digestion is needed for a different analytical technique. Examples are ultrapure materials, ultra-small amounts and biological tissues and fluids.

- Solid materials that are unique and should keep their integrity, such as materials from forensic studies and archaeological, cultural and art objects.

- Solid materials of which the bulk composition must be determined and for which surface or near-surface techniques such as XRF (X-ray fluorescence spectrometry) and some solidstate spectrometric techniques are inadequate.

\section{Final Remarks}

Primary methods of measurement, including neutron activation analysis, are based on physical-chemical principles with the highest metrological properties, can be completely described (in a measurement equation) and understood, and can have a complete uncertainty statement can be written down in terms of SI units.

A laboratory should only claim that it has the potential to operate a primary method of measurement when the entire measurement procedure is properly applied, and evidence thereof is available e.g. by successful participation in CCQM key-comparisons or pilot studies including the provision of complete uncertainty budgets. In addition, an analytical method can only be 'as good as the analyst'; this certainly applies to the primary methods of measurement as well.

\section{Acknowledgements}

The authors would like to thank R. M. Lindstrom, R. Zeisler, E. A. Mackey, M. A. Bacchi, and M. J. J. Koster-Ammerlaan for their valuable contributions contributing to the recognition of NAA as a primary method of measurement.

Received: August 3, 2009

[1] P. Bode, J. J. M. de Goeij, 'Activation Analysis', Encyclopedia of Environmental Analysis and Remediation, J. Wiley \& Sons, New York, 1998, ISBN 0-471-11708-0, pp 68-84.

[2] P. Bode, E. A. De Nadai Fernandes, R. R. Greenberg, J. Radioanal. Nucl. Chem. 2000, $245,109$.

[3] A primary method of measurement is a method having the highest metrological properties, whose operation can be completely described and understood, for which a complete uncertainty statement can be written down in terms of SI units. More specifically, a primary direct method was defined as a primary method of measurement that measures the value of an unknown without reference to a standard of the same quantity; and a primary ratio method as a primary method of measurement that measures the value of a ratio of an unknown to a standard of the same quantity; its operation must be completely described by a measurement equation.

[4] Report of the 2nd meeting of the CCQM, 1996.

[5] Report of the 5th Meeting (February 1998) of the CCQM, February 1998.

[6] M. J. T. Milton, T. J. Quinn, Metrologia 2001, $38,289$.

[7] Y. Aregbe, P. Taylor, CCQM K-24, 'Key comparison, Cadmium amount content in rice', Final report, IRMM, GE/R/IM/37/02/Sept. 2002.

[8] S. Noack, CCQM-K42, 'Key comparison, Determination of chromium, copper, iron, manganese and zinc in aluminium alloy', Final Report, BAM, June 2008.

[9] Report of the 13th meeting of the CCQM, 1920 April 2007. Report of the 14 th meeting of the CCQM, 3-4 April 2008

[10] R. R. Greenberg, R. M. Lindstrom, D. S. Simons, J. Radioanal. Nucl. Chem. 2000, 245 , 57.

[11] W. Tian, B. Ni, P. Wang, L. Cao, Y. Zhang, Accred. Qual. Assur. 2001, 6, 488

[12] R. R. Greenberg, J. Radioanal. Nucl. Chem. 2008, 278, 231. 